\title{
BMJ Open Implementation of a consumer-focused eHealth intervention for people with moderate-to-high cardiovascular disease risk: protocol for a mixed-methods process evaluation
}

Genevieve M Coorey, ${ }^{1,2}$ Lis Neubeck, ${ }^{3,4,5}$ Timothy Usherwood, ${ }^{1,2}$ David Peiris, ${ }^{1,2}$ Sharon Parker, ${ }^{6}$ Annie Y S Lau, ${ }^{7}$ Clara Chow, ${ }^{2,8}$ Kathryn Panaretto, ${ }^{9}$ Mark Harris, ${ }^{6}$ Nicholas Zwar, ${ }^{10}$ Julie Redfern ${ }^{2,8}$

To cite: Coorey GM, Neubeck L, Usherwood T, et al. Implementation of a consumer-focused eHealth intervention for people with moderate-to-high cardiovascular disease risk: protocol for a mixed-methods process evaluation. BMJ Open 2017;7:e014353. doi:10.1136/bmjopen-2016014353

- Prepublication history for this paper is available online. To view these files please visit the journal online (http://dx.doi.org/10.1136/ bmjopen-2016-014353).

Received 20 September 2016 Revised 7 December 2016 Accepted 9 December 2016

CrossMark

For numbered affiliations see end of article.

\section{Correspondence to} Genevieve Coorey; gcoorey@georgeinstitute. org.au

\section{ABSTRACT}

Introduction: Technology-mediated strategies have potential to engage patients in modifying unhealthy behaviour and improving medication adherence to reduce morbidity and mortality from cardiovascular disease (CVD). Furthermore, electronic tools offer a medium by which consumers can more actively navigate personal healthcare information.

Understanding how, why and among whom such strategies have an effect can help determine the requirements for implementing them at a scale. This paper aims to detail a process evaluation that will (1) assess implementation fidelity of a multicomponent eHealth intervention; (2) determine its effective features; (3) explore contextual factors influencing and maintaining user engagement; and (4) describe barriers, facilitators, preferences and acceptability of such interventions.

Methods and analysis: Mixed-methods sequential design to derive, examine, triangulate and report data from multiple sources. Quantitative data from 3 sources will help to inform both sampling and content framework for the qualitative data collection: (1) surveys of patients and general practitioners (GPs); (2) software analytics; (3) programme delivery records Qualitative data from interviews with patients and GPs, focus groups with patients and field notes taken by intervention delivery staff will be thematically analysed. Concurrent interview data collection and analysis will enable a thematic framework to evolve inductively and inform theory building, consistent with a realistic evaluation perspective. Eligible patients are those at moderate-to-high CVD risk who were randomised to the intervention arm of a randomised controlled trial of an eHealth intervention and are contactable at completion of the follow-up period; eligible GPs are the primary healthcare providers of these patients.

Ethics and dissemination: Ethics approval has been received from the University of Sydney Human Research Ethics Committee and the Aboriginal Health and Medical Research Council (AH\&MRC) of New South Wales. Results will be disseminated via scientific

\section{Strengths and limitations of this study}

- Evidence is growing that eHealth interventions are effective for supporting lifestyle behaviour change, medication adherence and engaging patients in healthcare navigation through shared record systems.

- In this project, we will use a mixed-methods approach to conduct a process evaluation of a randomised controlled trial (RCT) testing a consumer-focussed eHealth intervention for cardiovascular disease risk reduction, integrated with the primary healthcare electronic health record.

- Findings will contribute new knowledge about the important components for uptake, retention and impact; also factors affecting transferability to prevention strategies for other chronic diseases.

- Potential limitations are that some qualitative data will be collected before the RCT outcomes are known, and thus one or more factors influencing the trial results may be under-represented in these data.

forums including peer-reviewed publications and national and international conferences.

Trial registration number: ANZCTR 12613000715774.

\section{INTRODUCTION}

Cardiovascular disease (CVD) is a major global health problem and contributor to the wider public health epidemic of chronic diseases. ${ }^{1}$ Worldwide, CVD accounts for $48 \%$ of non-communicable disease mortality, with behavioural risk factors such as physical 
inactivity, poor dietary habits, tobacco use and medication non-adherence noted as key modifiable causes. ${ }^{2}{ }^{3}$ As the leading underlying cause of death for Australians, ${ }^{4}$ CVD is a national priority for disease prevention and healthcare cost reduction. ${ }^{5}$ CVD risk management is determined by the patient's overall or absolute CVD risk and the reduction of modifiable risk factors. ${ }^{6}$ Pharmacotherapy and lifestyle risk factor reduction decreases CVD morbidity and mortality, in primary prevention as well as in those with established CVD (secondary prevention) ${ }^{7}$ International data indicate that for those with established CVD, uptake of traditional secondary prevention programme approaches is typically low and only a minority attend an outpatient cardiac rehabilitation programme after hospital discharge. ${ }^{8-11}$ However, more than $80 \%$ of the Australian population visits a general practitioner (GP; synonymous here with the term 'primary care physician') at least once each year ${ }^{12}$ and more frequently as long-term health conditions necessitate. Therefore, the primary healthcare setting provides an opportunity for optimising reach of behaviour change counselling ${ }^{13}$ and is where eHealth approaches can complement clinician efforts to assist patients with awareness and responsibility for health behaviour modification. ${ }^{14} 15$

Technology-based approaches also fulfil broader national and international health system objectives to engage consumers in healthcare through the use of shared personal electronic records and decision-making support. ${ }^{16}$ These innovations are increasingly being recognised for their potential for more personalised care navigation that may engage consumers in health behaviour change. Such interventions offer alternative approaches to print-based or face-to-face formats for increasing access, uptake and engagement with effective GVD prevention. eHealth is defined as the use of information technologies to improve health, healthcare delivery and healthcare information systems. ${ }^{17} 18$ The success of such interventions has been reported in randomised controlled trials (RCTs) targeting specific behaviours, for example, increasing physical activity ${ }^{19}{ }^{20}$ and smoking cessation; ${ }^{21}{ }^{22}$ or targeting multifactorial aspects of lifestyle behaviour. ${ }^{23-25}$ In a description of the role of social cognitive theory in health promotion and disease prevention, Bandura ${ }^{26}$ suggests that using interactive technologies to first tailor communication about an individual's relevant personal factors, then to enable, motivate and guide, may enhance efforts to make lifestyle changes.

The Consumer Navigation of Electronic Cardiovascular Tools (CONNECT) RCT, has been described previously. ${ }^{27}$ It tests a multicomponent, tailored eHealth intervention to help patients adopt or increase healthy behaviours and medication adherence to improve CVD risk factor control. The primary end point is a composite of the proportion of patients whose blood pressure and fasting low-density lipoprotein cholesterol are meeting Australian guideline targets. The intervention was developed in a systematic user-centred design process previously described. ${ }^{28}$ A patient-focused web application, accessible via a mobile device or computer, is integrated with the primary healthcare electronic health record (EHR), enabling personalised risk factor data and interactive absolute CVD risk score calculation to be displayed, explained and updated via a visually engaging interface. Other elements include (1) interactive tools and information resources; (2) optional receipt of tailored healthy lifestyle tips and motivational messages; (3) interactive goal setting, tracking and virtual rewards; and (4) a social media/message board.

Complex health interventions have multiple interacting components. ${ }^{29}{ }^{30}$ Process evaluation can assist in identifying the critical elements, or combination of elements, from among multiple intervention components and any mediating or competing influences on their implementation. ${ }^{31-33}$ Moreover, a process evaluation collects data about programme delivery, receipt and setting which are essential to understanding the social processes that influence why a complex intervention does or does not have its intended impact. ${ }^{32} 34$ For this reason, process evaluations are increasingly reported in conjunction with RCT outcomes to explain impact and understand implications for future use of the intervention. ${ }^{35-38}$ Therefore, identifying how, for whom and in what contexts this type of intervention works will contribute new knowledge about the implementation of multicomponent, consumer-focussed eHealth interventions. In this paper, we describe the evaluation plan (table 1) for explaining programme process and effects, to assist with interpreting the trial outcomes and determining the important factors for programme scale up.

\section{Process evaluation aims}

1. Assess implementation fidelity in terms of intended content, reach, dose and duration of the intervention; and the role and extent of mediating factors on implementation fidelity;

2. Determine which features of the eHealth intervention function as effective triggers or opportunities for impact on health behaviour;

3. Explore contextual factors influencing and maintaining user engagement with the intervention;

4. Identify and describe barriers, facilitators, preferences and acceptability of an eHealth intervention from the perspective of patients and GPs.

\section{METHODS AND ANALYSIS Design}

A mixed-methods sequential design will be undertaken. ${ }^{40}$ Quantitative and qualitative data will be collected concurrently both during and at the end of the trial intervention period; however, analysis of some routinely collected quantitative data will precede patient sampling for qualitative data collection. ${ }^{41}$ Seven data sources will be used. Qualitative data collection and 
Objective

1. 1.1 Assess implementation fidelity ${ }^{32} 3339$ of the eHealth intervention with respect to the intended programme plan:

a. The intervention content is delivered in the intended manner and quality(Adherence to intervention concept)

b. Proportion of intended target audience that participates in all or part of the intervention

c. The amount of the intervention components that were provided to patients

d. How much of the activities and components was read, viewed or used for the intended duration? (Engagement of patients; see also 1.2(d) below)

e. For how long was the intervention implemented as intended by the trial design? (Related to intervention exposure)

1.2 Explain the role and extent of four factors that mediate implementation fidelity ${ }^{33}$

a. Intervention complexity

b. Facilitation strategies of programme delivery staff

c. Quality of programme delivery

d. Participant responsiveness

2. Determine the effective features of the intervention that function as triggers or opportunities for impact on health behaviour

3. Explore contextual factors influencing and maintaining user engagement with the intervention

4. Identify and describe barriers, facilitators, preferences and acceptability of an eHealth intervention from the perspective of patients and GPs

\section{Evaluation component}

\section{Fidelity}

measure

programme

providers

Function of

programme

users

scale up

Data source
Content

Reach

Dose delivered

Dose received/exposure

Frequency and duration

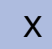

$-$

$X$

$-$
$\mathrm{X}$

- Moderating factors on the relationship between the intervention and its impact on recipients

- Patient characteristics

- Programme delivery factors

Patient characteristics

Personal beliefs and/or programme features as triggers for behaviour change action

- Personal circumstances or healthcare experiences affecting capacity to adopt new healthier behaviour

- Patient characteristics

- Perceived benefit and relevance of the intervention

- Personal circumstances or healthcare experiences affecting capacity to adopt or maintain healthier behaviour

- Intervention features used to adopt or increase healthier behaviour

- Patient characteristics

- Programme content and delivery factors

- Barriers and facilitators; relevance and acceptability of eHealth strategies
Programme delivery records Web programme analytics

Programme delivery records Web programme analytics Programme delivery records Web programme analytics Programme delivery records Web programme analytics

Programme delivery records

Programme delivery records Patient survey Focus groups

Programme delivery records Focus groups Patient interviews

Programme delivery records Focus groups

Patient survey

Patient interviews

Programme delivery records Focus groups

Patient survey

Patient interviews

GP interviews

GP survey 
reporting will be informed by the consolidated criteria for reporting qualitative research (COREQ) ${ }^{42}$ We will use the realistic evaluation framework ${ }^{43}$ to then describe how, why and among whom the intervention works in practice. In the realistic evaluation model, an intervention per se does not cause the outcomes observed; rather, one or more of its activities or components introduces an idea or motivation or opportunity (the mechanism) into a social or cultural situation (context), the combination of which may lead to an impact on behaviour (the observed outcome). ${ }^{44}$ In elucidating what might work for whom, how and in what circumstances, these concepts offer a fitting perspective for process evaluation because they focus less on the RCT effect between those exposed and not exposed to the intervention, and more on explaining context, mechanism and outcome within the exposed group. Therefore, these findings will inform intervention scale up and transferability because they increase understanding about characteristics of populations more likely to benefit.

The evaluation will be structured around the logic model outlined in figure 1. A logic model sets out the relationship between constructs of interest and mediating influences within a change process, namely the programme resource inputs, the activities or processes they produce, and the outputs that lead to the programme outcomes. ${ }^{45}{ }^{46}$ For the CONNECT intervention, the logic model depicts the intended inputs, activities, outputs and impact of the intervention as follows:
(1) resource inputs (the web application integrated with the EHR and the human resources required to implement it); (2) processes and activities of these inputs (the clinical and technical support from staff and the personalised and interactive features within the intervention; (3) intervention outputs (patient use both of intervention components and staff support options); and (4) impact on patients of exposure to the intervention (adoption of healthier lifestyle choices and more proactive engagement with the healthcare experience). Core characteristics of implementation fidelity (content, dose delivered, dose received) are shown as corresponding to specific sections of the logic model. Similarly, four mediating factors on fidelity (intervention complexity, facilitation strategies used by programme delivery staff, quality of delivery and patient responsiveness) ${ }^{33}$ are shown at their probable point(s) of influence. The relationship between these core characteristics and mediating factors will strengthen understanding about how programme effects happened-an important consideration in transferability and dissemination in other settings. ${ }^{32}$

\section{Participants}

Intervention arm patients and their GPs are eligible for participation in the process evaluation. Consenting patients (age $>18$ years) with established CVD or at moderate-to-high risk of a CVD event based on criteria outlined in the trial protocol will be included. ${ }^{27}$

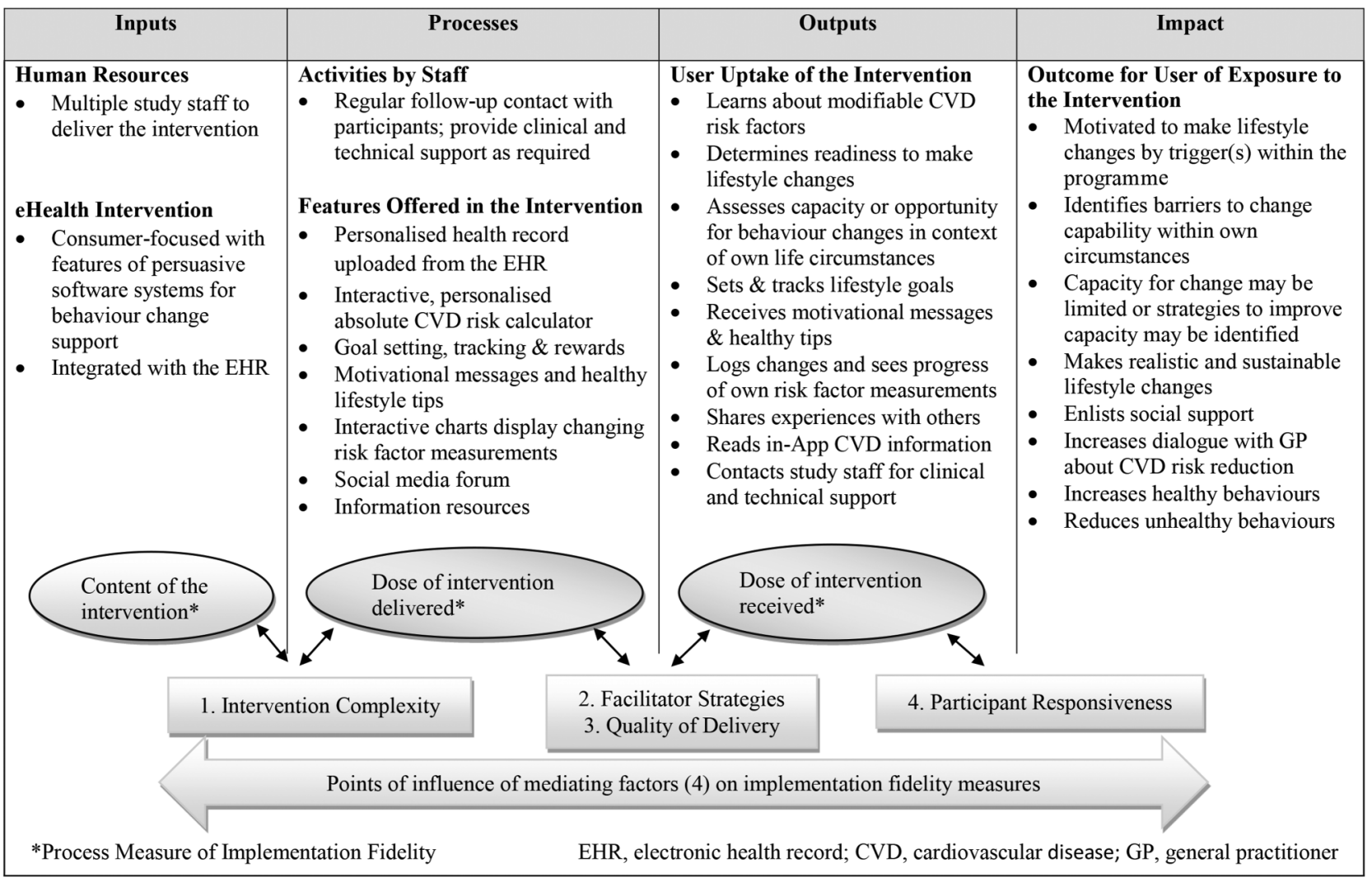

Figure 1 Logic model for Consumer Navigation of Electronic Cardiovascular Tools (CONNECT) implementation evaluation. 
Patients must be available in person or by telephone for the month 12 study follow-up visit and willing to provide written, informed consent to take part in either a focus group discussion or an interview (not both). Limiting patients to one format is intended to minimise responder burden and potentially duplicative conversations. Each format, however, has a distinct purpose in respect of data that is appropriate to group conversation versus personal or confidential topics. To avoid contaminating the intervention, patients will be invited to respond to the survey, and to take part in either a focus group or an interview, only after completion of the 12-month follow-up period. Consenting GPs will need to be the nominated primary healthcare provider for at least one RCT patient.

\section{Data sources}

\section{Web programme analytics}

Two methods will examine patients' direct interaction with the intervention. First, web page 'tagging' has been applied to the website to systematically record aspects of usage by patients during the study follow-up period. Tagging is a data source that logs user interaction with in-app features. It will provide real-time and historical figures about engagement with key interactive screens: absolute CVD risk calculator; counts of monthly personalised goal setting and goals achieved, and access of the message board/chat forum. These data enable researchers to identify whether portal login and use is sustained, declines or fluctuates over the timeline of follow-up. These metrics are independent of patients' self-reported use of the programme; they will both assist with sampling diverse patients for the qualitative data collection, and augment data from the patient surveys and focus groups to inform understanding of programme appeal and attrition. Separately, a customised tracker counts the motivational and healthy lifestyle tips sent to patients by email and/or short message service. Since patients can opt out of receiving these messages, these data will help describe the interest in this feature of the intervention. Second, data about the number of unique monthly website login sessions on three device types (laptop computer, tablet or Smartphone) will be obtained from a commercial web analytics service (Google Analytics).

\section{Programme delivery records}

Database records maintained by study staff record the number of intervention arm patients who were trained to use the eHealth programme (informing programme reach); any facilitation strategies that enabled patients to more easily use the intervention (maximising reach and dose received); and the content, duration and format of scheduled and ad hoc communication during the follow-up period (considerations for intervention duration, dose received and staff skill mix needs). Feedback offered by patients during communication with staff is categorised and quoted (anonymously), providing additional contextual data. The nature of technical or content errors in a patient's app that are identified and fixed prior to training or during follow-up is recorded, ensuring that intended content within the app, for example, data imported from the EHR, is correct (maximising dose delivered). As indicated, these records both reflect programme delivery quality and will complement data about implementation fidelity, namely: (1) proportion of intended patients who actually took part (reach); (2) the extent of patient uptake of the intervention (dose received); (3) the extent that all components of the intervention were delivered as frequently and for as long as planned (dose delivered and duration), ${ }^{33} 39$ and (4) intervention delivery time requirements (important for resource needs assessment in sustaining or upscaling such an intervention).

\section{Survey of patients}

Eligible patients will be invited to complete a short survey at the final study follow-up visit, thereby minimising recall bias. Patients will be asked to complete their survey in confidence; the survey will be mailed to those unable to attend the month 12 study visit in person. The two-page survey includes 10 statements with Likert scale responses about use of various features of the intervention (such as goal setting and tracking, receipt of motivational lifestyle tips, and charting weight or other measurements), and effect on healthy behaviours (such as weekly physical activity, eating habits, medication adherence); six questions have categorical responses about ease and frequency of use of the intervention and access to study staff for support; and three questions allow free-text responses about programme utility and preferred features/screens. Our previous work in testing the concept and design of the intervention ${ }^{28}$ informed the choice of content for which feedback is sought; general guidelines for questionnaire design were then used to develop a reliable survey. ${ }^{47}$ It was reviewed for content validity by the multidisciplinary research team (nurses, physiotherapist, GPs) with expertise in instrument design and familiarity with the intervention.

\section{Focus group discussions}

Eligible patients will be invited by telephone, postal or email invitation to take part in focus groups of $\sim 8-10$ people per group. A minimum of three focus groups will be conducted at locally available or participating health service facilities for $\sim 1$ hour duration per group. We will use the software analytics data to enable a diversity sampling approach to the mix of patients in terms of age, sex, CVD risk status and frequency of intervention usage, the latter metric being of particular interest as a variable associated with clinical outcome. Recruitment for focus groups will be consecutive until no new themes or categories emerge (thematic saturation); however, it is anticipated that at least 25-30 people will be invited to take part. Standard focus group methods will be used including facilitation by a trained health professional 
with knowledge of the RCT, a non-participant observer/ note-taker, setting of ground rules and audio recording. ${ }^{48}$ A discussion guide will expand on key feedback themes from the patient survey, including usability and use of the intervention, perceived quality of delivery and programme support; preference for duration of programme participation; potential improvements or changes to the intervention components; and important or relevant features that impacted behaviour or changed how the patient engaged with their GP or other healthcare services regarding their care. The emphasis of focus groups is therefore on feedback about practical implementation issues that are appropriate to a group conversation, rather than targeted to personal health information or circumstances.

\section{Interviews with patients}

Eligible patients will be invited by telephone, postal or email invitation to take part in a one-on-one interview of up to 1 hour duration. Interviews will explore personal and potentially sensitive topics that are inappropriate for a focus group format. The researcher will ask the interviewee about his/her responses to the content and options offered within the eHealth intervention; their subsequent choices about making lifestyle-related changes, and their capacity for action within their personal circumstances. Within the realistic evaluation model, the purpose of interviews is to use the patient's experience of the programme to test a hypothesis about how and why intervention components create an opportunity for behaviour change; and from these responses build understanding about the characteristics and contexts of users for whom this happens. Interviews with programme users are the key data source in constructing data within this framework. ${ }^{43}$ The researcher thus proposes a 'theory' about programme mechanisms acting in the patient's personal context or circumstances to cause an impact/outcome, and seeks the interviewee's refinement of this proposal, by falsifying or confirming the ideas through telling their own story. ${ }^{43}{ }^{49}$ Important differences in contextual factors, for example, socioeconomic status, risk factor awareness, lifestyle and social support that affect decision-making and promote or hinder programme uptake may therefore be identified. Data within the routinely collected software metrics will assist us using a maximum variation sampling method based on patient demographics, CVD risk status, and the type and frequency of use of intervention features. Sampling will continue until thematic saturation is achieved. Interviews will be conducted at locally available or participating health service facilities or general practices; at the George Institute or via telephone, as convenient for the patient. A semistructured interview guide will be used by a trained health professional to conduct the interview and audio recording will ensure important verbal data are captured. Notes may be made by the researcher after the interview to document relevant nonverbal information.

\section{Survey of GPS}

All GPs taking part in the RCT will be invited by mail, email and/or direct phone contact to complete a survey at the end of the study. The aim is to obtain feedback about their experience of the RCT set-up and conduct in their workplace, and of using the software required to facilitate the shared health record innovation. Also of interest are their usual strategies for lifestyle modification counselling for their patients with moderate or high CVD risk, and their perception of relevance and benefit of eHealth approaches. The two-page survey of 19 questions will include 6 questions requiring Likert scale responses (related to research participation); 2 allowing multiple response selection (related to research participation and to lifestyle counselling preferences); 5 with categorical responses (related to programme content and impact on their patients); and 6 allowing free-text comments (related to perceptions of benefit and drawbacks). Content targets feedback agreed by the research team to be important to future dissemination of an eHealth strategy that is integrated with GP medical record software systems. General guidelines for questionnaire design were then used to develop a reliable survey. ${ }^{47}$ It was reviewed for content validity by the multidisciplinary research team (nurses, physiotherapist, GPs) with expertise in instrument design and understanding of the general practice environment. The survey will be sent by email, or postal mail with a return addressed envelope, and telephone follow-up will ensure maximum number of surveys are returned.

\section{Interviews with GPs}

GPs participating in the RCT will be invited by email or postal mail to take part in a confidential one-on-one interview at the end of the study. The purpose of interviews is to: expand on themes within the survey so as to triangulate these data; explore their previous and current experiences with eHealth strategies; gain insights about patient characteristics affecting choices made about behaviour change support; describe perceived benefits, barriers or concerns from using the integrated health record software, or from GP-patient interactions about the intervention's content or impact. Combining the interview data with those from the feedback surveys will enable a richer GP perspective on programme utility, equity, barriers and likelihood of adoption. Given that linkage with the primary healthcare software is central to this intervention, these data will influence appraisal of sustaining and scaling up such a strategy. We anticipate that interviews with a consecutive sample of $\sim 10$ participating GPs from different suburban locations reflecting diverse patient demography will be sufficient; however, we will continue to recruit until we achieve thematic saturation. A trained healthcare professional will conduct and audio record the interviews of $\sim 30 \mathrm{~min}$ duration at the practice or health service, or via telephone, as convenient for the GP. A discussion guide of open-ended questions will be 
used. Notes may be made by the researcher after an in-person interview to document relevant non-verbal information.

\section{Data analysis}

The website server logs will be analysed for frequency of logins by patients and number of visits to specific pages; also the number and delivery format of lifestyle message tips. Program analytics data will be presented as frequencies and proportions to enable description of user engagement with the intervention generally and with specific interactive features, for example, social media forum and goal tracking. Process measures (reach, dose and duration of the intervention) will be analysed as proportions, frequencies and means. Subgroup analyses will assess for any differential impact of the intervention on RCT outcome measures by extent of uptake of the intervention. Statistical significance will be assessed using $\chi^{2}$ tests for categorical variables and t-tests for continuous variables. Univariate and multivariate regression models will be built to determine associations between various exposure variables and the prespecified trial outcomes. Descriptive statistics will be derived from the survey responses and will be reported as frequencies and proportions; for example, the type and extent of engagement with key intervention features; likes and dislikes about the programme; perceived impact of, and overall views about, the role of an EHR-integrated intervention to support CVD risk factor reduction, and so on.

Feedback from patients within telephone and email communication during the study follow-up period will be categorised and quotations noted. These add to the programme feedback from survey and focus groups data and offer insight into characteristics of patients for whom the intervention did or did not appeal. For the focus group and interview data, a minimum of two researchers will independently conduct thematic analysis of transcripts. Using the constant comparison method, codes will be identified inductively based on emergent themes. ${ }^{50}$ Reporting of these data will be guided by the COREQ guidelines. ${ }^{42}$ An inductive approach will also be taken to analysis of any textual responses within the patient and GP surveys, and in the records of patient contact with programme staff. Concurrent interview data collection and analysis will enable a thematic framework to evolve inductively and help inform theory building about the intervention from a realistic evaluation perspective. Integration of qualitative and quantitative analyses will thus occur at multiple phases in the evaluation, from sampling of patient interviewees, for example, to the broader analysis and interpretation. Results from our different data sources will be integrated and interpreted to improve validity of conclusions. We will use tools from recognised implementation frameworks 5152 to support our analyses and reporting of the data. Particular emphasis will be placed on any divergent findings that arise. ${ }^{41}$

\section{ETHICS AND DISSEMINATION}

Clinical Trial Agreements are signed between participating primary healthcare services and the George Institute for Global Health, Australia. Patients and GPs who are invited to participate in a focus group or interview will be provided with an information sheet explaining its purpose and conduct and asked to provide written informed consent before taking part. Results of this research will be disseminated via scientific forums including peer-reviewed publications and presentations at national and international conferences.

\section{DISCUSSION}

Two key process questions for a complex health intervention are to understand its mechanisms of impact and the context(s) in which the impact occurs. ${ }^{29}$ This process evaluation plan addresses these questions for a technology-based intervention designed to influence patient attitudes and/or behaviour in respect of lifestyle-related CVD risk factors and navigation of their wider healthcare experience. Realistic evaluation is a framework with which to examine complex programmes in these terms. Furthermore, explaining programme process and effects assists in interpreting the trial outcomes and determining the important factors for programme scale up and dissemination.

Multiple dimensions of engagement with an eHealth intervention are therefore important to characterise: behavioural (what the person does); cognitive (what the person knows and understands) and emotional (what the person feels about their disease and steps to manage it). ${ }^{53}$ No single metric describes website engagement. Page visits, time spent and interactive components used are relatively accessible measures. Little is known, however, about user characteristics that influence engagement in respect of the above three dimensions for an intervention such as this one, and which may inform understanding about the programme components that drive ongoing participation versus foreseeable programme attrition. In turn, the relationship between programme engagement and user impact may illuminate the threshold level of involvement that confers a benefit to patients-the assumption being that website use at best fluctuates, but diminishes over time. ${ }^{55}$ This process evaluation will explore these questions of meaning, social context and characteristics of those for whom the intervention was or was not helpful. Further, survey and qualitative data will address usability, overall user experience and social validity that gauge consumer acceptance of web-based interventions. ${ }^{56}$

The questions of interest described in this research concern fidelity of implementation, how and for whom the intervention works, and thus what influences future scale up, expansion or transferability of such a programme. We have therefore incorporated selected reporting criteria from the Reach, Effectiveness, Adoption, Implementation, and Maintenance (RE-AIM) evaluation 
framework $^{51}$ as apply to this study, although the research is not explicitly modelled on this. Each of the above five dimensions within RE-AIM comprise multiple reporting criteria which previous studies have reported to varying degrees, often to the exclusion of qualitative methods. ${ }^{57}$ We are targeting pertinent criteria within the dimensions of reach, effectiveness and implementation; for example, percentage of individuals who take part $(\mathrm{R})$, qualitative data to understand lifestyle behaviour change outcomes (E), and programme delivery measures (I), respectively.

Mixed-methods data collection is a methodological strength for exploring process questions within the RCT because both qualitative and quantitative data from patients and GPs will enable richer complementary insights than from either method alone. ${ }^{41} 50$ A systematic mapping review of qualitative inquiry within RCTs into aspects of intervention delivery underscored the advantage both to interpreting trial findings and improving external validity. ${ }^{58} \mathrm{~A}$ potential limitation of this evaluation process is that data from surveys, focus groups and interviews may be subject to recall bias when obtained after 12 months of study follow-up; we did not undertake formal validity and reliability testing of the surveys. Adherence is likely to have at best fluctuated over that period; thus, recall bias may favour the later over the earlier months of using the intervention. Also, without prior knowledge of the RCT outcome, some uncertainty is possible about the content or topics on which to focus aspects of the data collection that precede trial completion; however, analysis of these data will likely occur when trial outcome data are available. Timing the process evaluation data collection ahead of RCT outcome analysis may also risk that unanticipated trial outcomes will be underrecognised in the process data. ${ }^{34}$ On the other hand, the many intervention components reflect principles of persuasive software system design and social cognitive theory; therefore, combining these development influences with the concepts explored in realistic evaluation enables this evaluation to have a more defined than speculative focus.

\section{CONCLUSION}

Evidence is growing that eHealth interventions are effective for improving lifestyle behaviours associated with development and progression of chronic diseases. At-risk patients and their primary healthcare providers are key to our understanding about the role of these innovative approaches in primary and secondary CVD prevention. Expansion of eHealth as a medium for public health interventions can benefit from reporting of how they interact both with contextual factors and any possible moderating influences of their component features or delivery methods. ${ }^{59}{ }^{60}$ A complex eHealth intervention designed for health behaviour change support is best understood by process evaluation research about programme fidelity (how the intervention delivery compared with the intended protocol); and how, and for whom, the intervention triggers intent and action for behaviour change within the recipient's circumstances and care experience (the mechanisms and context explained by realistic evaluation). Taken together, these process data will expand and enrich understanding of RCT results and may inform transferability to prevention programmes for other chronic conditions in which lifestyle-related factors drive disease risk.

\section{Author affiliations}

${ }^{1}$ The George Institute for Global Health, Sydney, New South Wales, Australia ${ }^{2}$ Sydney Medical School, University of Sydney, Sydney, New South Wales, Australia

${ }^{3}$ School of Health and Social Care, Edinburgh Napier University, Edinburgh, UK

${ }^{4}$ Sydney Nursing School, Charles Perkins Centre, University of Sydney, Sydney, New South Wales, Australia

${ }^{5}$ Faculty of Medicine, Nursing and Health Sciences, School of Nursing \& Midwifery, Flinders University, Adelaide, Australia

${ }^{6}$ Centre for Primary Health Care and Equity, University of New South Wales, Sydney, New South Wales, Australia

${ }^{7}$ Centre for Health Informatics, Australian Institute of Health Innovation,

Macquarie University, Sydney, New South Wales, Australia

${ }^{8}$ Cardiovascular Division, The George Institute for Global Health, Sydney, New South Wales, Australia

${ }^{9}$ Centre for Chronic Disease, School of Medicine, University of Queensland,

Brisbane, Queensland, Australia

${ }^{10}$ School of Public Health and Community Medicine, University of New South Wales, Sydney, New South Wales, Australia

Twitter Follow Lis Neubeck @lisneubeck, Julie Redfern @jredheart and The George Institute for Global Health @georgeinstitute

Acknowledgements The authors acknowledge members of the Steering Committee responsible for the design and development of the CONNECT RCT who are not co-authors on this paper, namely Professor E Coiera, Associate Professor N Hayman, Dr E Heeley, Associate Professor S Jan and Professor A Rodgers. Also acknowledged are members of the project team responsible for implementing the trial: J Mulley, C Pitt, J Forbes and T Nguyen.

Contributors GC led the drafting of all sections of the manuscript; JR, LN and TU provided important feedback on the initial draft. Each author substantially contributed to design and concept of the programme process evaluation, provided critical revisions of important intellectual content and approved the final version for publication.

Funding The study is funded by the National Health and Medical Research Council (NHMRC; grant number 1047508). GC is funded by a University of Sydney Postgraduate Award (SC0649). JR is funded by a National Health and Medical Research Council Career Development Fellowship (1061793) co-funded with a National Heart Foundation Future Leader Fellowship (G160523). CC is funded by a NHMRC Career Development Fellowship (1105447) co-funded by a National Heart Foundation Future Leader Fellowship (100808). AL is funded by the NHMRC Centre of Research Excellence in eHealth (1032664)

Competing interests None declared.

Ethics approval Ethics approval has been received from the University of Sydney Human Research Ethics Committee (ID 2013/716) and the Aboriginal Health and Medical Research Council (AH\&MRC) of New South Wales (ID 959/13).

Provenance and peer review Not commissioned; externally peer reviewed.

Open Access This is an Open Access article distributed in accordance with the Creative Commons Attribution Non Commercial (CC BY-NC 4.0) license, which permits others to distribute, remix, adapt, build upon this work noncommercially, and license their derivative works on different terms, provided the original work is properly cited and the use is non-commercial. See: http:// creativecommons.org/licenses/by-nc/4.0/ 


\section{REFERENCES}

1. Yeates K, Lohfeld L, Sleeth J, et al. A global perspective on cardiovascular disease in vulnerable populations. Can J Cardiol 2015;31:1081-93.

2. World Health Organisation. Global status report on noncommunicable diseases 2010. http://www.who.int/nmh/ publications/ncd_report2010/en/ (accessed Apr 2016).

3. Bansilal S, Castellano JM, Fuster V. Global burden of CVD: focus on secondary prevention of cardiovascular disease. Int $J$ Cardiol 2015;201(Suppl 1):S1-7.

4. Australian Institute of Health and Welfare. Leading Causes of Death, 2013. http://www.aihw.gov.au/deaths/leading-causes-of-death/ (accessed Apr 2016)

5. National Preventative Health Taskforce. Taking preventative actiona response to Australia: the healthiest country by 2020-the Report of the National Preventative Health Taskforce 2010. http://www. preventativehealth.org.au/internet/preventativehealth/publishing.nsf/ Content/6B7B17659424FBE5CA25772000095458/\$File/tpa.pdf (accessed Apr 2016)

6. National Vascular Disease Prevention Alliance. Guidelines for the management of absolute cardiovascular disease risk. 2012. https:// heartfoundation.org.au/images/uploads/publications/

Absolute-CVD-Risk-Full-Guidelines.pdf (accessed Aug 2016).

7. Unal B, Critchley JA, Capewell S. Modelling the decline in coronary heart disease deaths in England and Wales, 1981-2000: comparing contributions from primary prevention and secondary prevention. BMJ 2005;331:614

8. Redfern J, Hyun K, Chew DP, et al. Prescription of secondary prevention medications, lifestyle advice, and referral to rehabilitation among acute coronary syndrome inpatients: results from a large prospective audit in Australia and New Zealand. Heart2014;100:1281-8.

9. Thomas RJ, Miller NH, Lamendola C, et al. National survey on gender differences in cardiac rehabilitation programs. Patient characteristics and enrollment patterns. J Cardiopulm Rehabil 1996;16:402-12.

10. Ruano-Ravina A, Pena-Gil C, Abu-Assi E, et al. Participation and adherence to cardiac rehabilitation programs. A systematic review. Int J Cardiol 2016;223:436-43.

11. Suaya JA, Shepard DS, Normand SL, et al. Use of cardiac rehabilitation by Medicare beneficiaries after myocardial infarction or coronary bypass surgery. Circulation 2007;116:1653-62.

12. Australian Bureau of Statistics. Patient experiences in Australia: summary of findings. 2014. http://www.abs.gov.au/ausstats/abs@ . nsf/Lookup/4839.0main+features32012-13 (accessed May 2016).

13. The Royal Australian College of General Practitioners. Guidelines for preventive activities in general practice. 8th edn. East Melbourne, VIC: The Royal Australian College of General Practitioners, 2012:50-1.

14. Talboom-Kamp EP, Verdijk NA, Harmans LM, et al. An eHealth platform to manage chronic disease in primary care: an innovative approach. Interact J Med Res 2016;5:e5.

15. Carey M, Noble N, Mansfield E, et al. The role of eHealth in optimizing preventive care in the primary care setting. J Med Internet Res 2015;17:e126.

16. Institute of Medicine. Health IT and patient safety: building safer systems for better care. Washington DC: The National Academies Press, 2012:115-19.

17. Burke LE, Ma J, Azar KM, et al. Current science on consumer use of mobile health for cardiovascular disease prevention: a scientific statement from the American Heart Association. Circulation 2015;132:1157-213.

18. Alkhaldi B, Sahama T, Huxley C, et al. Barriers to implementing eHealth: a multi-dimensional perspective. Stud Health Technol Inform 2014;205:875-9.

19. Foster C, Richards J, Thorogood M, et al. Remote and web 2.0 interventions for promoting physical activity. Cochrane Database Syst Rev 2013;(9):CD010395.

20. Wanner M, Martin-Diener E, Braun-Fahrlander C, et al. Effectiveness of active-online, an individually tailored physical activity intervention, in a real-life setting: randomized controlled trial. J Med Internet Res 2009;11:e23.

21. Strecher VJ, McClure JB, Alexander GL, et al. Web-based smoking-cessation programs: results of a randomized trial. Am J Prev Med 2008;34:373-81.

22. Brendryen HF, Drozd F, Kraft P. A digital smoking cessation program delivered through internet and cell phone without nicotine replacement (happy ending): randomized controlled trial. J Med Internet Res 2008;10:e51.

23. Bennett GG, Herring SJ, Puleo E, et al. Web-based weight loss in primary care: a randomized controlled trial. Obesity (Silver Spring) 2010;18:308-13.
24. Hutchesson MJ, Rollo ME, Krukowski R, et al. eHealth interventions for the prevention and treatment of overweight and obesity in adults: a systematic review with meta-analysis. Obes Rev 2015;16:376-92.

25. Chow CK, Redfern J, Hillis GS, et al. Effect of lifestyle-focused text messaging on risk factor modification in patients with coronary heart disease: a randomized clinical trial. JAMA 2015;314:1255-63.

26. Bandura A. Health promotion by social cognitive means. Health Educ Behav 2004;31:143-64.

27. Redfern J, Usherwood T, Harris $\mathrm{M}$, et al. A randomised controlled trial of a consumer-focused e-health strategy for cardiovascular risk management in primary care: the consumer navigation of electronic cardiovascular tools (CONNECT) study protocol. BMJ Open 2014;4: e004523.

28. Neubeck L, Coorey G, Peiris D, et al. Development of an integrated e-health tool for people with, or at high risk of, cardiovascular disease: the consumer navigation of electronic cardiovascular tools (CONNECT) web application. Int J Med Inform 2016;96:24-37.

29. Moore GF, Audrey S, Barker M, et al. Process evaluation of complex interventions: Medical Research Council guidance. BMJ 2015;350: h1258.

30. Campbell M, Fitzpatrick R, Haines A, et al. Framework for design and evaluation of complex interventions to improve health. BMJ 2000;321:694-6.

31. McGraw SA, Sellers DE, Stone EJ, et al. Using process data to explain outcomes: an illustration from the child and adolescent trial for cardiovascular health (CATCH). Eval Rev 1996;20:291-312.

32. Hasson H. Systematic evaluation of implementation fidelity of complex interventions in health and social care. Implement Sci 2010;5:67

33. Carroll C, Patterson M, Wood S, et al. A conceptual framework for implementation fidelity. Implement Sci 2007;2:40.

34. Munro A, Bloor M. Process evaluation: the new miracle ingredient in public health research? Qual Res 2010;10:699-713.

35. Jago R, Rawlins E, Kipping RR, et al. Lessons learned from the AFLY5 RCT process evaluation: implications for the design of physical activity and nutrition interventions in schools. BMC Public Health 2015;15:946.

36. Edwards MJ, May T, Kesten JM, et al. Lessons learnt from the Bristol Girls Dance Project cluster RCT: implications for designing and implementing after-school physical activity interventions. BMJ Open 2016:6:e010036.

37. Leamy M, Clarke E, Le Boutillier C, et al. Implementing a complex intervention to support personal recovery: a qualitative study nested within a cluster randomised controlled trial. PLOS ONE 2014;9:e97091.

38. Wood F, Salam A, Singh K, et al. Process evaluation of the impact and acceptability of a polypill for prevention of cardiovascular disease. BMJ Open 2015;5:e008018.

39. Steckler A, Linnan L. Process evaluation for public health interventions and research. San Francisco, CA: Jossey-Bass, 2002:11-17.

40. Creswell JW. Research design. 2nd edn. Thousand Oaks, CA: Sage Publications, 2003:208-20.

41. Creswell JW, Plano-Clark V. Designing and conducting mixed methods research. Thousand Oaks, CA: Sage Publications, 2007:84-5.

42. Tong A, Sainsbury $P$, Craig J. Consolidated criteria for reporting qualitative research (COREQ): a 32-item checklist for interviews and focus groups. Int J Qual Health Care 2007;19:349-57.

43. Pawson R, Tilley N. Realistic evaluation. London, UK: Sage Publications, 1997.

44. Astbury B, Leeuw FL. Unpacking black boxes: mechanisms and theory building in evaluation. Am J Eval 2010;31:363-81.

45. West JF. Public health program planning logic model for community engaged Type 2 diabetes management and prevention. Eval Program Plann 2014;42:43-9.

46. Saunders RP, Evans MH, Joshi P. Developing a process-evaluation plan for assessing health promotion program implementation: a how-to guide. Health Promot Pract 2005;6:134-47.

47. Boynton PM, Greenhalgh T. Selecting, designing, and developing your questionnaire. BMJ 2004;328:1312-15.

48. Barbour R. Doing focus groups. London, UK: Sage Publications, 2007:75-91.

49. Manzano A. The craft of interviewing in realist evaluation. Evaluation 2016;22:342-60.

50. Curry LA, Nembhard IM, Bradley EH. Qualitative and mixed methods provide unique contributions to outcomes research. Circulation 2009;119:1442-52.

51. Glasgow RE, Vogt TM, Boles SM. Evaluating the public health impact of health promotion interventions: the RE-AIM framework. Am J Public Health 1999;89:1322-7.

52. Damschroder LJ, Aron DC, Keith RE, et al. Fostering implementation of health services research findings into practice: 
a consolidated framework for advancing implementation science. Implement Sci 2009;4:50.

53. Barak A, Klein B, Proudfoot JG. Defining internet-supported therapeutic interventions. Ann Behav Med 2009;38:4-17.

54. Barello S, Triberti S, Graffigna G, et al. eHealth for patient engagement: a systematic review. Front Psychol 2015;6:2013.

55. Glasgow RE, Christiansen SM, Kurz D, et al. Engagement in a diabetes self-management website: usage patterns and generalizability of program use. J Med Internet Res 2011;13:e9.

56. Danaher BG, Seeley JR. Methodological issues in research on web-based behavioral interventions. Ann Behav Med 2009;38:28-39.
57. Gaglio B, Shoup JA, Glasgow RE. The RE-AIM framework: a systematic review of use over time. Am J Public Health 2013;103: e38-46.

58. O'Cathain A, Thomas KJ, Drabble SJ. What can qualitative research do for randomised controlled trials? A systematic mapping review. BMJ Open 2013;3:e002889.

59. Rychetnik L, Frommer M, Hawe $\mathrm{P}$, et al. Criteria for evaluating evidence on public health interventions. J Epidemiol Community Health 2002;56:119-27.

60. Glasgow RE, Phillips SM, Sanchez MA. Implementation science approaches for integrating eHealth research into practice and policy. Int J Med Inform 2014;83:e1-11. 